\title{
Accounting for Item Response Process and Response Styles Using the Unfolding Item
} Response Tree (UIRTree) Model

\author{
Zhaojun $\mathrm{Li}^{1}$, Bo Zhang ${ }^{2}$, Mengyang $\mathrm{Cao}^{3}$, Louis Tay ${ }^{4}$ \\ ${ }^{1}$ Department of Psychology, The Ohio State University \\ ${ }^{2}$ Department of Psychological and Brain Sciences, Texas A \& M University \\ ${ }^{3}$ Independent Researcher \\ ${ }^{4}$ Department of Psychology, Purdue University
}

Draft version 1. This paper is under review. Please do not copy or cite without the authors' permission.

Correspondence concerning this article should be addressed to Zhaojun Li, Department of Psychology, The Ohio State University, 240C Lazenby Hall, 1827 Neil Avenue, Columbus, OH 43210. Email: 1i.8282@,osu.edu 


\begin{abstract}
Many researchers have found that unfolding models may better represent how respondents answer Liker-type items and response styles (RSs) often have moderate to strong presence in responses to such items. However, the two research lines have been growing largely in parallel. The present study proposed an unfolding item response tree (UIRTree) model that can account for unfolding response process and RSs simultaneously. An empirical illustration showed that the UIRTree model could fit a personality dataset well and produced more reasonable parameter estimates. Strong presence of the extreme response style (ERS) was also revealed by the UIRTree model. We further conducted a Monte Carlo simulation study to examine the performance of the UIRTree model compared to three other models for Likert-scale responses: the Samejima's graded response model, the generalized graded unfolding model, and the dominance item response tree (DIRTree) model. Results showed that when data followed unfolding response process and contained the ERS, the AIC was able to select the UIRTree model, while BIC was biased towards the DIRTree model in many conditions. In addition, model parameters in the UIRTree model could be accurately recovered under realistic conditions, and wrongly assuming the item response process or ignoring RSs was detrimental to the estimation of key parameters. In general, the UIRTree model is expected to help in better understanding of responses to Liker-type items theoretically and contribute to better scale development practically. Future studies on multi-trait UIRTree models and UIRTree models accounting for different types of RSs are expected.
\end{abstract}

Keywords. unfolding response process, response style, generalized graded unfolding model, unfolding item response tree model 


\section{Accounting for Item Response Process and Response Styles Using the Unfolding Item Response Tree (UIRTree) Model}

As more and more studies have found that non-cognitive variables like personality and vocational interests are robust predictors of important life outcomes such as job performance, academic achievement, and subjective well-being (e.g., Anglim et al., 2020; Judge et al., 2013; Nye et al., 2012, 2021; Poropat, 2009), non-cognitive assessment has become increasingly popular in many contexts. For example, personality tests are widely used for personnel selection (Feintzeig, 2015). The Organization for Economic Co-operation and Development (OECD) also included personality and vocational interest as a core assessment component for the Programme for the International Assessment of Adult Competencies (OECD, 2018).

Non-cognitive assessments often rely on Likert scales in which respondents are asked to indicate their degree of agreement with items. Responses are then subjected to models like Samejima's Graded Response Model (SGRM; Samejima, 1969) to estimate respondents' standings on the latent trait continuum. The legitimacy of using models like SGRM is contingent upon two assumptions: (1) respondents follow a dominance response process such that there is a monotonically increasing relationship between the latent trait level and the probability of endorsing the item, and (2) responses to items within a scale is systematically influenced by the focal trait. However, past studies suggest that both assumptions may not be tenable. For example, regarding the first assumption, it has been shown that the unfolding response process (that assumes an inverted U-shaped relationship between the probability of endorsing an item and a respondent's latent trait level) better represents how individuals respond to items measuring non-cognitive constructs (Cao et al., 2015; Drasgow et al., 2010; Tay et al., 2009; Sun et al., 2021; Zhang et al., 2020). As for the second assumption, numerous studies have shown that there are stable individual differences in response styles (RSs) -- the idiosyncratic use of response options regardless of item content and their levels of latent trait 
(Wetzel et al., 2016). It means that the same observed score on an item may represent different levels of trait for different individuals because some individuals are more likely to use extreme response options, while others are more likely to use nonextreme options, rendering scores less comparable across individuals. Failure to address either assumption will result in biased estimates of item and person parameters, which can have severe consequences for scale development, scoring, and selection outcomes.

Many efforts have been devoted to resolving issues of the response process and RSs. For example, Roberts and colleagues (2000) developed the Generalized Graded Unfolding Model (GGUM) to better model the unfolding response process, which has been successfully applied to many non-cognitive assessments. Further, item response tree (IRTree) models have been proposed and well-received as a flexible tool for handling RSs (e.g., Böckenholt, 2012, 2017, 2019; Henninger \& Meiser, 2020; Jeon \& De Boeck, 2016; Lang et al., 2019; Lang \& Tay, 2019; Lievens et al., 2018; Sun et al., 2021). However, the two lines of research have been developing in parallel, and researchers concerned about the response process often overlook the issue of RSs, and vice versa. There is a need to integrate the two lines of research to more optimally assess non-cognitive constructs.

Therefore, the present study presents an unfolding item response tree (UIRTree) model that simultaneously accounts for RSs and the unfolding response process for non-cognitive assessment. Specifically, Study 1 used an empirical dataset to reveal the magnitude of extreme response style (ERS) and the presence of unfolding response process, as well as the (costs) benefits of (not) modeling them. Study 2 adopted a simulation design to systematically examine the statistical performance of the UIRTree model and the power of AIC and BIC for accurate model selection. A user-friendly tutorial on how to fit the model in the $\mathrm{R}$ package mirt (Chalmers, 2012) is also provided. To the best of our knowledge, this is the first attempt to combine the IRTree model and the unfolding model. 


\section{Unfolding response process and the GGUM}

Two distinct types of models have been proposed to describe how individuals respond to Likert-type items. Dominance models assume that the probability of a stronger endorsement of an item increases monotonically as their trait level increases. Though often not explicitly stated, most commonly used psychometric models belong to dominance models (e.g., Confirmatory Factor Analysis, Rasch model, 2-Parameter Logistic Model [2PLM], and SGRM). Dominance models are well suited for tests measuring "maximal behaviors" that challenge the limits of individual's capacity, such as cognitive or psychomotor tests (Tay \& Drasgow, 2012; Tay \& Ng, 2018). One of the most common dominance models is the SGRM with the model equation:

$$
P\left(Y_{i} \geq k \mid \theta_{j}\right)=\frac{\exp \left(\alpha_{i} \theta_{j}-\delta_{i k}\right)}{1+\exp \left(\alpha_{i} \theta_{j}-\delta_{i k}\right)}
$$

where $Y_{i}$ is the subjective response to item $i, \theta_{j}$ is the location of individual $j$ on the latent continuum, $\alpha_{i}$ is the discrimination of item $i, \delta_{i k}$ is the difficulty parameter (also called category threshold parameter) of item $i$ to achieve a category no smaller than $k$.

For measures that assess "self-reported typical thoughts and behaviors," such as personality, respondents tend to first gauge the degree of match between their true levels of thoughts/behaviors and the levels of thoughts/behaviors conveyed by the item (or the distance between the item location and the respondent's latent trait level). The closer the item content matches the respondent's true self, the higher the probability that the respondent will endorse the item. For example, the item "I am as extraverted as an average person" describes an average level of extraversion. A very extraverted or introverted individual is less likely to endorse this item as the degree of extraversion conveyed by this item does not match that of the respondents. Moderately extraverted individuals are more likely to endorse this item as there is a close match. Therefore, unfolding models are more appropriate for the assessment of typical behaviors (Tay $\& \mathrm{Ng}, 2018$ ). Figure 1 demonstrates the key difference between dominance and unfolding 
models. Adopting the appropriate measurement model aligned with the response process underling the target construct is a fundamental issue for measurement validity (Borsboom et al., 2004).

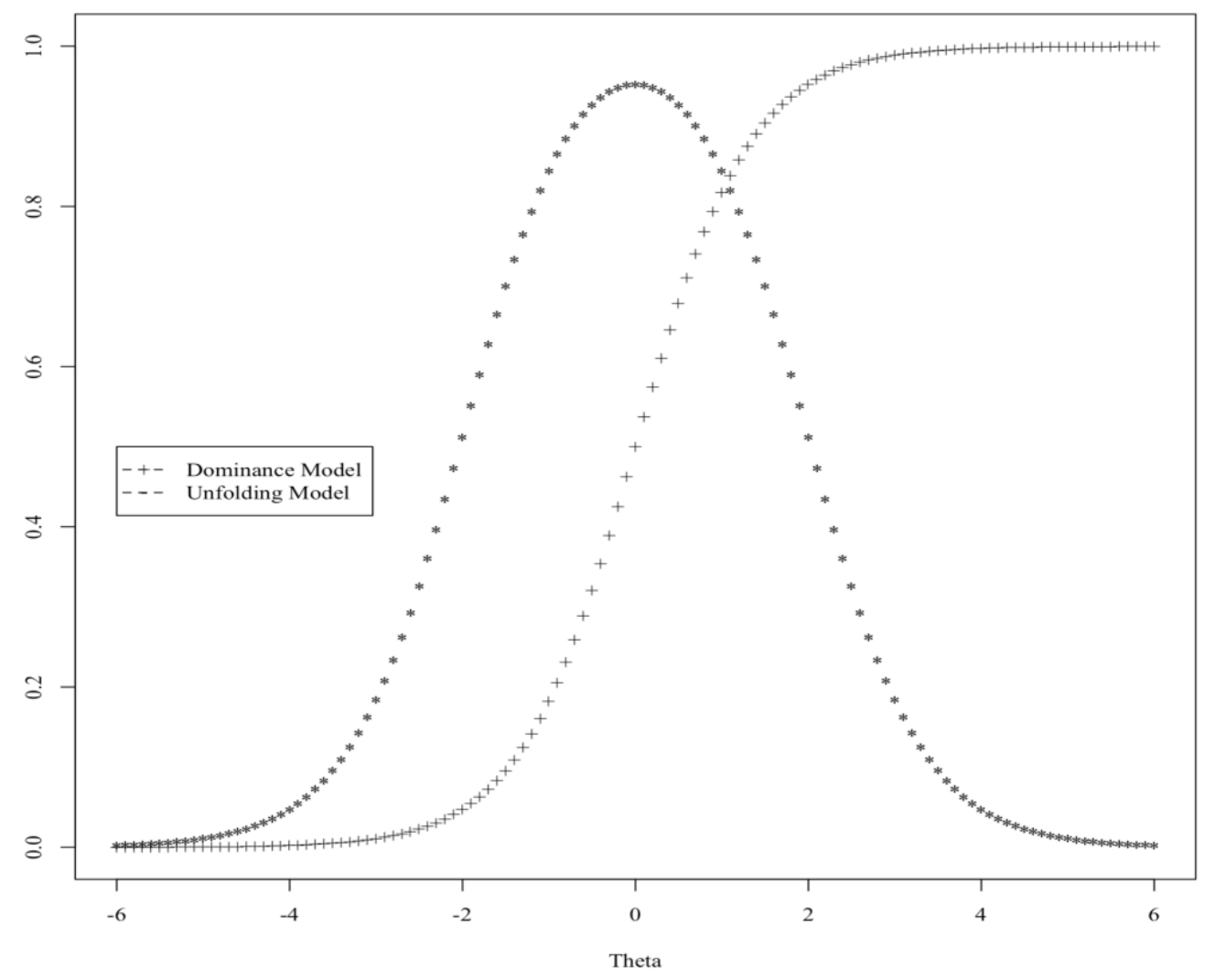

Figure 1. Item response curves for unfolding and dominance models

Among the unfolding models, GGUM is no doubt the most widely used one due to 1) its capability to describe the unfolding process, 2) ease of interpretation, and 3) the availability of free software for parameter estimation. According to Roberts et al. (2000), the GGUM assumes that an observed response can result from two possible subjective responses. Each of the two subjective responses corresponds to an individual's position either above or below the item's location on the latent continuum. As a result, an item with categories $z=0,1,2, \ldots, C$ would correspond to subjective responses $y=0,1,2, \ldots, M$, where $M=2 C+1$. All subjective responses follow a generalized partial credit model (Muraki, 1992) with the model equation: 


$$
P\left(Y_{i}=y \mid \theta_{j}\right)=\frac{\exp \left\{\alpha_{i}\left[y\left(\theta_{j}-\delta_{i}\right)-\sum_{k=0}^{y} \tau_{i k}\right]\right\}}{\sum_{w=0}^{M}\left\{\exp \left\{\alpha_{i}\left[w\left(\theta_{j}-\delta_{i}\right)-\sum_{k=0}^{w} \tau_{i k}\right]\right\}\right\}}
$$

where $Y_{i}$ is the subjective response to item $i, \theta_{j}$ is the location of individual $j$ on the latent continuum, $\alpha_{i}$ is the discrimination of item $i, \delta_{i}$ is the location of item $i$ on the latent continuum, and $\tau_{i k}$ is the threshold of the $k^{t h}$ option for item $i$. To establish model identifiability, a model constraint is set that $\sum_{k=0}^{w} \tau_{i k}=0$. The model equation for the observed response to time $i, Z_{i}$, is then defined as:

$$
\begin{aligned}
P\left(Z_{i}=z \mid \theta_{j}\right) & =P\left(Y_{i}=z \mid \theta_{j}\right)+P\left(Y_{i}=W-z \mid \theta_{j}\right) \\
& =\frac{\exp \left\{\alpha_{i}\left[z\left(\theta_{j}-\delta_{i}\right)-\sum_{k=0}^{Z} \tau_{i k}\right]\right\}+\exp \left\{\alpha_{i}\left[(M-z)\left(\theta_{j}-\delta_{i}\right)-\sum_{k=0}^{z} \tau_{i k}\right]\right\}}{\sum_{w=0}^{C}\left\{\exp \left\{\alpha_{i}\left[w\left(\theta_{j}-\delta_{i}\right)-\sum_{k=0}^{w} \tau_{i k}\right]\right\}+\exp \left\{\alpha_{i}\left[(M-w)\left(\theta_{j}-\delta_{i}\right)-\sum_{k=0}^{w} \tau_{i k}\right]\right\}\right.} .
\end{aligned}
$$

Over the past two decades, GGUM has been shown to provide a better fit than dominance models to data of personality (Chernyshenko et al., 2007; Carter et al., 2014; Drasgow et al., 2012), interests (Tay et al., 2009), job satisfaction (Carter \& Dalal, 2010), emotional intelligence (Cho et al., 2015), attachment style (Sun et al., 2021), interpersonal values (Ling et al., 2016), and attitude (Roberts et al., 1999). Mis-specifying an unfolding model as a dominance model will lead to biased item parameter estimates (e.g., truly wellperforming items showing low discrimination power) and adversely impact item selection. It will also result in distorted rankings of individuals, especially for those at the two ends of the trait continuum, thus hampering the quality of selection decision (Stark et al., 2006), reducing criterion-related validity (Sun et al., 2021), and lowering the power to detect curvilinear effects (Cao et al., 2018; Carter et al., 2017).

\section{Response styles and the IRTree model}

Response styles (RSs) refer to tendencies to choose response options in systematic but content-irrelevant ways (Baumgartner \& Steenkamp, 2001). For example, extreme response style (ERS) is the tendency to choose extreme options. It has been consistently demonstrated that RSs exist in non-cognitive measurements (Batchelor \& Miao, 2016; Baumgartner \& 
Steenkamp, 2001; Rorer, 1965), and the presence of RSs may distort the measurement of the construct of interest, which leads to estimation bias and threatens measurement validity (Eid \& Rauber, 2000; Johnson \& Bolt, 2010; McDaniel et al., 2011; Moors, 2004; Plieninger, 2017).

Among the many approaches to handling RSs, the IRTree model (Böckenholt, 2012; De Boeck \& Partchev, 2012) exhibits its great advantages of disassociating the focal trait and RSs while measuring them simultaneously. Evidence from many empirical studies shows that different types of RSs can be well addressed by the IRTree model (i.e., Ames \& Myers, 2020; De Boeck \& Partchev, 2012; Jeon \& De Boeck, 2019; Zettler et al., 2016). Since IRTree models in previous studies all assumed a dominance response process, we term such IRTree models as dominance IRTree (DIRTree) models in contrast to the proposed UIRTree model in the rest of this article.

In the DIRTree model, an item with polytomous responses is decomposed into several sub-items given a tree structure where the sub-items are represented by nodes, and the item categories are represented by leaves (i.e., end-nodes). In RS analyses with the DIRTree model, items are decomposed into focal trait-related and RS-related sub-items. In this way, both the focal trait and RSs can be measured by their respective sub-items without being influenced by each other, and their latent correlations can be easily estimated. For instance, let us consider a four-point item with categories $0,1,2$ and 3 corresponding to "strongly disagree," "disagree," "agree," and "strongly agree," respectively. We can use an IRTree structure to separate the item into three binary sub-items that are to measure whether an observed response is negative (i.e., category 0 or 1 ) or positive (i.e., category 2 or 3 ); if negative, whether it is extreme (i.e., category 0 ) or not (i.e., category 1 ); if positive, whether it is extreme (i.e., category 3 ) or not (i.e., category 2). Furthermore, it is reasonable to assume that the first sub-item measures the focal trait while the last two sub-items reflect the ERS. In this way, we are able to fit a twofactor item response model with the first sub-item loading on the focal trait and the last two 
sub-items loadings on the ERS. If we adopt the 2PLM for the first sub-item and the Rasch model (Rasch, 1960) for the last two sub-items, the model equations in the DIRTree model are:

$$
\begin{aligned}
& P\left(U_{1 i}=1 \mid \zeta_{j}\right)=\frac{\exp \left(\alpha_{i} \theta_{j}-\delta_{i}\right)}{1+\exp \left(\alpha_{i} \theta_{j}-\delta_{i}\right)}, \\
& P\left(U_{2 i}=1 \mid \zeta_{j}\right)=\frac{\exp \left(\zeta_{j}-\beta_{2 i}\right)}{1+\exp \left(\zeta_{j}-\beta_{2 i}\right)} \\
& P\left(U_{3 i}=1 \mid \zeta_{j}\right)=\frac{\exp \left(\zeta_{j}-\beta_{3 i}\right)}{1+\exp \left(\zeta_{j}-\beta_{3 i}\right)}
\end{aligned}
$$

where $U_{n i}$ is the response to the $n^{\text {th }}$ sub-item of item $i, \theta_{j}$ is the location of individual $j$ on the continuum of the focal trait, $\alpha_{i}$ is the discrimination parameter on the focal trait, $\delta_{i}$ is the location of the first sub-item of item $i$ on the focal trait, $\zeta_{j}$ is the location of individual $j$ on the continuum of the ERS, $\beta_{2 i}$ is the location of the second sub-item of item $i$ on the continuum of the ERS, and $\beta_{3 i}$ is the location of the third sub-item of item $i$ on the continuum of the ERS. Accordingly, the probability for each observed response to item $i, U_{i}$, can be defined as follows:

$$
\begin{aligned}
& P\left(U_{i}=0 \mid \theta_{j}, \zeta_{j}\right)=P\left(U_{1 i}=0 \mid \theta_{j}\right) P\left(U_{2 i}=1 \mid \zeta_{j}\right), \\
& P\left(U_{i}=1 \mid \theta_{j}, \zeta_{j}\right)=P\left(U_{1 i}=0 \mid \theta_{j}\right) P\left(U_{2 i}=0 \mid \zeta_{j}\right), \\
& P\left(U_{i}=2 \mid \theta_{j}, \zeta_{j}\right)=P\left(U_{1 i}=1 \mid \theta_{j}\right) P\left(U_{3 i}=0 \mid \zeta_{j}\right), \\
& P\left(U_{i}=3 \mid \theta_{j}, \zeta_{j}\right)=P\left(U_{1 i}=1 \mid \theta_{j}\right) P\left(U_{3 i}=1 \mid \zeta_{j}\right) .
\end{aligned}
$$

\section{The UIRTree Model and Its Estimation}

Despite the fruitful development in DIRTree models and unfolding models, the two lines of research have not crossed paths yet. As noted above, it is equally important to address issues of RSs and unfolding responding to achieve better measurement validity and more accurate estimates of item and item parameters. Therefore, we take the first step to join the two lines of research and propose the UIRTree model.

Similar to the DIRTree model, the UIRTree model also decomposes items with polytomous responses into different nodes representing different sub-items. The major 
difference between the two models lies in how we model the sub-item that corresponds to the focal trait (e.g., "disagree" vs "agree"). As Equation 4 shows, the DIRTree model adopts a 2PL model assuming that respondents with a higher level of higher latent trait are more likely to "agree." In contrast, the UIRTree model assumes an unfolding response process for the node representing the focal trait and adopts GGUM for it. Take the aforementioned four-point item as an example. After being decomposed, the first sub-item representing the focal trait is fitted by GGUM with the model equation:

$$
P\left(U_{1 i}=1 \mid \theta_{j}\right)=\frac{\exp \left\{\alpha_{i}\left[\left(\theta_{j}-\delta_{i}\right)-\tau_{i}\right]\right\}+\exp \left\{\alpha_{i}\left[2\left(\theta_{j}-\delta_{i}\right)-\tau_{i}\right]\right\}}{1+\exp \left\{\alpha_{i}\left[3\left(\theta_{j}-\delta_{i}\right)-\tau_{i}\right]\right\}+\exp \left\{\alpha_{i}\left[\left(\theta_{j}-\delta_{i}\right)-\tau_{i}\right]\right\}+\exp \left\{\alpha_{i}\left[2\left(\theta_{j}-\delta_{i}\right)-\tau_{i}\right]\right\}},
$$

where $\delta_{i}$ is the location of the first sub-item of item $i$ on the continuum of the focal trait, and the meanings of the item parameters $\alpha_{i}, \delta_{i}$, and $\tau_{i}$ are the same as in Equations 2 and 3 . The second and third sub-items representing the ERS can be fitted by any dichotomous dominance models. If the Rasch model is adopted, then the model equations for the two sub-items are the same as Equations 5-6 in the UIRTree model. Lastly, the same as for the DIRTree model, the model equations of the observed response to item $i, U_{i}$, are Equations 7-10 in the UIRTree model.

The IRTree structure of the UIRTree model for four-point items is presented in Figure 2. It should be noted that, just like the DIRTree model, the UIRTree model represents a general framework and can be easily extended in multiple ways to test different hypotheses. For example, we can replace the Rasch model for the last two sub-items with more complex models such as the 2PL model and use model fit comparison to select the best-fitting model. In addition, responses can also be decomposed following different tree structures to explore other types of RSs. Multidimensionality of focal traits can also be easily handled. We provided coding schemas for different tree structures and corresponding R scripts for estimation in the tutorial. 


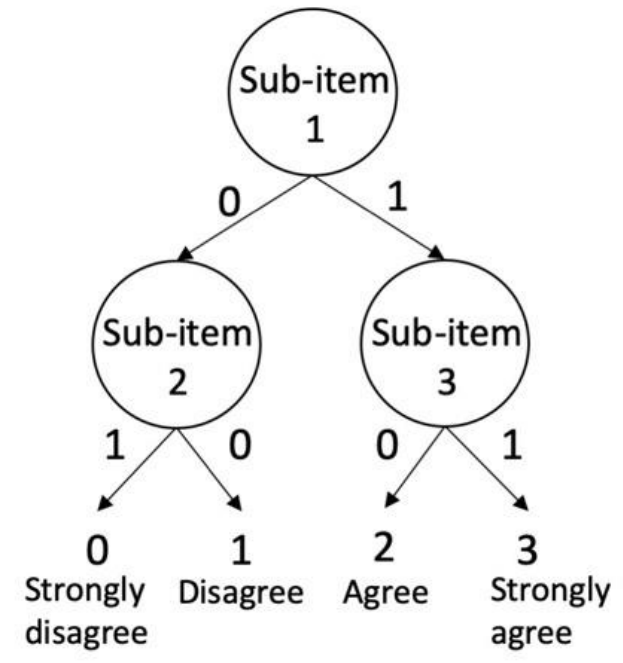

Figure 2. IRTree structure of the UIRTree model for four-point items. Circles represent sub-items. The first sub-item is estimated with the GGUM and the last two sub-items are estimated with the Rasch model. The four leaves of the tree structure represent the four item categories.

DIRTree models can be easily fitted using many latent variable modeling software programs that can deal with categorical data (e.g., Mplus, lavaan). However, the estimation of UIRTree model is trickier. Most latent variable modeling software programs are designed for dominance models, and software programs for unfolding models like GGUM2004 (Roberts et al., 2006) or R packages GGUM (Tendeiro \& Castro-Alvarez, 2019) and bmggum (Tu et al., 2021) can only handle unfolding models. Fortunately, the R package mirt (Chalmers, 2012) is capable of dealing with both dominance and unfolding models. Specifically, mirt allows the specification of hybrid models where a subset of items follow dominance models and the other subset of items follow unfolding models. Estimation of such hybrid models is what we need for the UIRTree models because sub-items related to focal traits need to be fitted by GGUM and sub-items related to RSs need to be fitted by dominance models. Another feature of mirt that makes it particularly suitable for the UIRTree model is that researchers can easily specify priors for any parameters. Marginal maximum likelihood (MML) estimation of GGUM is 
known for issues of convergence and extreme estimates (e.g., $\delta_{e s t}=30$ or $\left.\delta_{s e}=100\right)$, especially for scales with a smaller number of response categories. Incorporating priors into the estimation process (marginal maximum a posterior; MMAP) can greatly stabilize the estimation process and provide reasonable estimates without sacrificing computation efficiency (Roberts \& Thompson, 2011). Additionally, various model/person fit indices, scoring methods, and plotting functions are readily available in mirt such that users can easily carry out many postestimation analyses. Therefore, we chose mirt as the estimation engine for the UIRTree model.

It should be noted that Javaras and Ripley (2007) and Liu and Wang (2018) also developed two non-IRTree models to handle the unfolding response process and RSs simultaneously. Despite all the merits of these two models, they are still limited in the following ways. First, Javaras and Ripley (2007) operationalized RSs as an individual difference in thresholds and did not specify the type(s) of RSs that is accounted for. The conceptual ambiguity of RSs in this model may make it less useful when researchers are interested in the nature of a specific type of RS (e.g., stability and correlates of the ERS). Second, Liu and Wang (2018) constrained threshold parameters in their model to be the same across items to achieve stable estimates. Such constraints may be hard to justify theoretically. Moreover, both models have to be estimated by specific software that is not very accessible to substantive researchers. The UIRTree model is intended as an alternative model that shares the same logic as the popular DIRTree model, is more intuitive and user-friendly, and overcomes some limitations of the two earlier models.

\section{The Present Study}

Utilizing an empirical personality dataset that included extreme and intermediate items, Study 1 aims to demonstrate the necessity of accounting for RSs and the unfolding response process by examining (1) the strength of the ERS (2) the type of response process, and (3) the consequences of failing to adopt the UIRTree model. Having shown the necessity of the 
UIRTree model, Study 2 used Monte Carlo simulation to systematically examine the statistical performance of the UIRTree model and whether information criteria (AIC \& BIC) can effectively select the correct model.

\section{Study 1. Empirical Illustration}

\section{Datasets}

A published personality dataset was reanalyzed, where researchers measured the orderliness, dominance, and curiosity facets of personality (Cao et al., 2015). The authors included 4 positively worded items, 4 negatively worded items, and 12 intermediate items for each construct. Each item was rated on a four-point scale ("strongly disagree," "disagree," "agree," and "strongly agree"). The reason why we chose this dataset is that they included extreme and intermediate items, which are critical for revealing the unfolding response process. The dataset has not been analyzed using the DIRTree or UIRTree model before. Cronbach's $\alpha$ s of the three facets were $0.84,0.77$, and $0.87^{1}$. There were 355 undergraduates from a large Midwestern university in the United States in total. Among them, $71 \%$ were female. There were $70.1 \%$ White, $15.2 \%$ Asian, 5.9\% Hispanic or Latino, 5.6\% Black or African American, and $3.1 \%$ others.

\section{Analytic details}

In order to thoroughly examine both the presence of ERS and the type of response process, we chose four candidate models: the SGRM (assuming no ERS and a dominance response process; Equation 1), the GGUM (assuming no ERS and an unfolding response process; Equation 3), the DIRTree model (assuming ERS and a dominance response process; Equations 4-10), and the UIRTree model (assuming ERS and an unfolding response process;

\footnotetext{
${ }^{1}$ We note that the computation of Cronbach's $\alpha$ is also (implicitly) based on the dominance assumption. Technically speaking, Cronbach's $\alpha$ is not appropriate for unfolding models. Here we presented thess numbers just in case some readers may be interested.
} 
Equations 5-10 and 11). All models were fitted to each facet of the dataset using the R package mirt 1.33.2 (Chalmers, 2012) with the MMAP estimator. In all models, the focal trait was specified to follow a standard normal distribution with a mean of zero and a variance of one. In the DIRTree and UIRTree models, a Rasch model was adopted for ERS such that the mean of ERS was fixed to be zero while its variance and its correlation with the focal trait were freely estimated. To stabilize the estimation of the UIRTree model, a $\log N(0.2,0.5)$ prior was set for the discrimination parameters $\alpha_{i}$, and a $N(0,3)$ prior was set for the location parameters $\delta_{i}$. No priors were set for other parameters. As MMAP is a full-information estimator, missing data was automatically handled. The datasets and associated $\mathrm{R}$ scripts can be found in the online supplementary material.

\section{Results}

\section{Model comparisons}

AIC and BIC were used to compare the relative model fit of the four candidate models. Across all three facets, it was consistent that the UIRTree model outperformed the GGUM and the DIRTree model outperformed the SGRM with respect to AIC and BIC, indicating that it is necessary to model ERS. As for the comparison between the UIRTree model and the DIRTree model, AIC and BIC suggested different results. Specifically, two out of three facets had smaller AIC when estimated with the UIRTree model versus the DIRTree model. In comparison, all three facets had smaller BIC when estimated with the DIRTree model versus the UIRTree model. Therefore, whether the items followed the dominance response process or the unfolding response process remained unclear, especially given that the discrimination power of AIC and BIC has never been investigated in such scenarios before. 
Table 1

Model fit in the empirical study

\begin{tabular}{lllll}
\hline & \multicolumn{3}{l}{ Estimation model } \\
\cline { 2 - 5 } & SGRM & GGUM & $\begin{array}{l}\text { DIRTree } \\
\text { model }\end{array}$ & $\begin{array}{l}\text { UIRTree } \\
\text { model }\end{array}$ \\
\hline AIC & & & & \\
Orderliness & 13069.62 & 13165.66 & $\mathbf{1 2 8 9 8 . 6 9}$ & 12955.07 \\
Dominance & 11894.33 & 11902.19 & 11821.87 & $\mathbf{1 1 7 8 5 . 0 1}$ \\
Curiosity & 13615.12 & 13548.35 & 13539.01 & $\mathbf{1 3 4 2 7 . 9 6}$ \\
BIC & & & & \\
Orderliness & 13379.39 & 13552.87 & $\mathbf{1 3 2 1 6 . 2}$ & 13427.47 \\
Dominance & 12200.23 & 12285.53 & $\mathbf{1 2 1 3 5 . 5 1}$ & 12253.53 \\
Curiosity & 13924.89 & 13935.56 & $\mathbf{1 3 8 5 6 . 5 2}$ & 13900.36 \\
\hline
\end{tabular}

Note. The smallest AIC and BIC values per row are bolded. 


\section{Estimates of the ERS}

Across the three facets in the dataset, the variances of the ERS were estimated to be $1.62,2.89$, and 1.58 in the UIRTree model and $1.61,2.92$, and 1.58 in the DIRTree model, suggesting that ERS had a strong impact on item responses.

The absolute values of estimated latent correlations between focal traits and ERS were $.33, .33$, and .25 in the UIRTree model, and were $.31, .34$, and .28 in the DIRTree model. The weak to moderate correlation estimates indicated that focal traits and ERS were well differentiated. We also calculated the correlation between ERS factor scores derived from the three facets. It can be seen from Table 2 that these factor scores were moderately to strongly correlated with each other, with correlations ranging from .54 to .59 . The omega coefficient based on the one-factor model was .79 for both the UIRTree and the DIRTree models, suggesting the existence of a strong general ERS factor. Taken together, these results cogently showed that (1) ERS has a strong presence, (2) ERS is distinct from focal traits, and (3) there is a strong general ERS factor across scales. Therefore, it is important to model ERS.

\section{Table 2}

Correlations among ERS factor scores

\begin{tabular}{lccc}
\hline & 1 & 2 & 3 \\
\hline Orderliness & -- & .59 & .55 \\
Dominance & .59 & -- & .54 \\
Curiosity & .54 & .54 & -- \\
\hline
\end{tabular}

Note. Values below the diagonal were from the UIRTree model and those above the diagonal were from the DIRTree.

\section{Key parameter estimates}

Having shown the presence of a strong ERS factor, we further compared a common set of estimated parameters from the GGUM and the UIRTree model to examine the benefits of UIRTree model. Specifically, both models provided information about the discrimination 
power $(\alpha)$ and location $(\delta)$ of each item on the focal trait. These parameters are key to item selection during the scale development stage. For example, scale developers often aim for a set of items with high discrimination power and varying location parameters for better measurement (Tay \& $\mathrm{Ng}, 2018$ ). Items that do not meet these criteria are often removed from further consideration.

Several interesting patterns emerged. First, item discrimination parameters $\alpha$ on the focal trait obtained from the UIRTree model tended to be higher compared to those from the GGUM, indicating that the ERS may mask the discrimination power of items and make psychometrically sound items look unsatisfactory ${ }^{2}$. Second, $\delta$ estimates from the UIRTree model for most intermediate items - those predicted to have close-to-zero $\delta s-$ were close to zero and showed clear unfolding while many of these parameters from the GGUM deviated substantially from zero. As expected, the positively and negatively worded items had more extreme location estimates than intermediate items in both models. The close correspondence between theoretical prediction and empirical estimates provides strong support for the validity of the UIRTree model.

\section{Focal trait factor scores}

We further looked into factor scores of the focal traits to examine the impact of the ERS. The empirical reliability estimates of factor scores of the focal traits in the four estimation models were shown in the diagonal of Table 3. It is expected that the focal trait scores had relatively lower empirical reliability in the two IRTree models as four data points were contributing to the estimation of focal trait scores in the SGRM and GGUM, while only two data points contributed in the UIRTree and the DIRTree models. Whereas, in comparison to

\footnotetext{
${ }^{2}$ Similarly, it was found that the DIRTree model in general had higher item discrimination parameters on the focal trait compared to the SGRM, which further provided evidence of the masking effect of the ERS.
} 
the DIRTree model, the UIRTree model had clearly better empirical reliability across the three facets, which indicated that the estimates from the UIRTree model were more reliable.

Table 3

Correlation among focal factor scores and empirical reliability (diagonal) from different models

\begin{tabular}{|c|c|c|c|c|c|c|c|c|c|c|c|c|c|}
\hline Construct & Model & 1 & 2 & 3 & 4 & 5 & 6 & 7 & 8 & 9 & 10 & 11 & 12 \\
\hline \multirow{4}{*}{ Orderliness } & SGRM & .85 & & & & & & & & & & & \\
\hline & GGUM & .98 & .85 & & & & & & & & & & \\
\hline & DIRTree & .88 & .87 & .73 & & & & & & & & & \\
\hline & UIRTree & .85 & .88 & .97 & .76 & & & & & & & & \\
\hline \multirow{4}{*}{ Dominance } & SGRM & .36 & .35 & .30 & .30 & .93 & & & & & & & \\
\hline & GGUM & .36 & .35 & .31 & .30 & .99 & .93 & & & & & & \\
\hline & DIRTree & .31 & .31 & .32 & .32 & .89 & .89 & .80 & & & & & \\
\hline & UIRTree & .28 & .27 & .30 & .28 & .87 & .89 & .94 & .85 & & & & \\
\hline \multirow{4}{*}{ Curiosity } & SGRM & .12 & .11 & .09 & .09 & .23 & .21 & .19 & .19 & .89 & & & \\
\hline & GGUM & .11 & .10 & .09 & .09 & .21 & .19 & .17 & .18 & .98 & .90 & & \\
\hline & DIRTree & .06 & .06 & .07 & .07 & .23 & .21 & .20 & .20 & .93 & .93 & .83 & \\
\hline & UIRTree & .09 & .07 & .10 & .08 & .20 & .19 & .17 & .21 & .90 & .92 & .94 & .87 \\
\hline
\end{tabular}

Note. Correlations among factor scores for the same focal trait from the four estimation models and correlations among factor scores of the three focal traits within each estimation model are bolded. Empirical reliability estimates are presented in the diagonal.

We also calculated the correlations between factor scores of the same personality facet derived from the four estimation models to examine the impact of ERS on factor score estimation. As shown below the diagonal of Table 3, the correlations between focal-trait factor scores of the models that accounted for ERS (i.e., the DIRTree model and the UIRTree model) and the models that did not account for ERS (i.e., the SGRM and the GGUM) were from .85 to .93 . While being high, these correlations were still clearly below one, indicating that ERS had a non-negligible impact on trait score estimation. Furthermore, scatter plots in Figure 3 showed that focal-trait factor scores of respondents with extreme values were impacted the most by the ERS. It is expected since extreme responses were completely accounted for the 
focal trait and brought about extreme factor scores in the models without ERS, whereas they were explained by the ERS in the models with ERS.

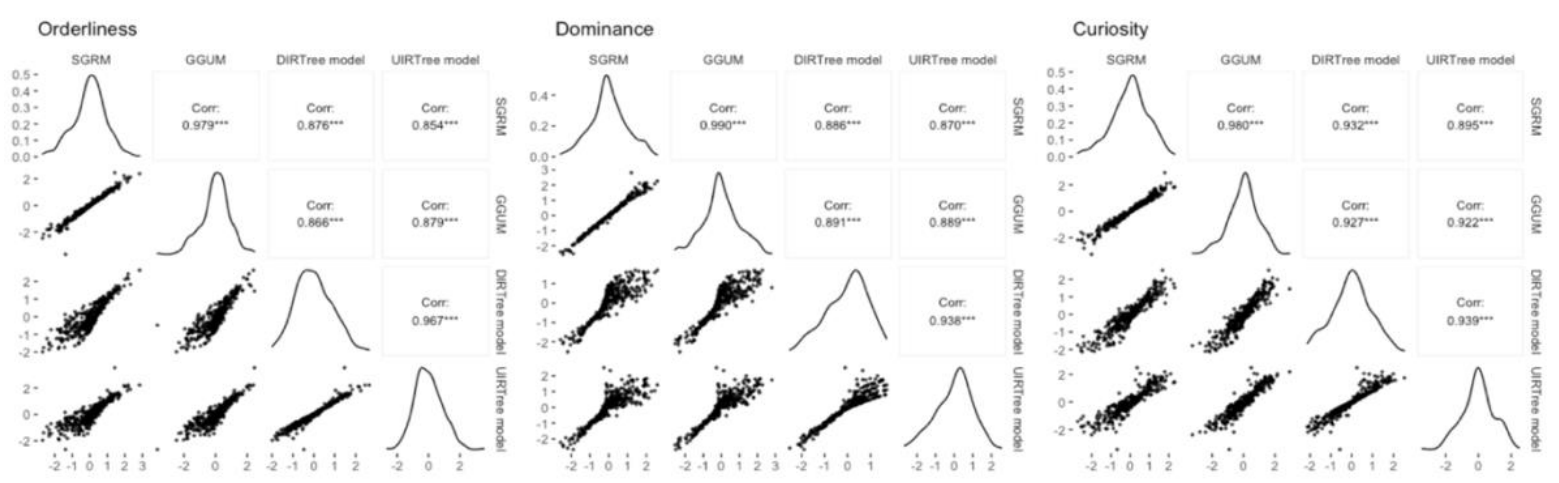

Figure 3. Scatter plots of focal-trait factor scores from different estimation models

\section{Discussion}

Using an empirical dataset, we found that (1) AIC and BIC consistently indicated that the two IRTree models fitted the data better than the SGRM and the GGUM, though less consistent evidence was found for the differentiation between the DIRTree and UIRTree models; (2) ERS had a strong presence in the item responses across scales; (3) the UIRTree model provided more reasonable item parameter estimates (i.e., higher discrimination parameters for most items and less extreme location parameters for intermediate items) that were more aligned with theory compared to the GGUM; and (4) unmodeled ERS impacted the rank order of individuals and inflated correlations among distinct facets. The results provided preliminary support for the validity of the UIRTree model. However, two key questions remained to be answered before we can confidently embrace the UIRTree model. First, the DIRTree model and the UIRTree model could not be clearly differentiated by AIC and BIC, which leads to a question about the discrimination power (i.e., the power to correctly select the model that describes the data best) of AIC and BIC in terms of model selection. Second, it is also important to determine how accurately parameters can be recovered for the UIRTree model and what factors will impact the estimation accuracy. A Monte Carlo simulation study is needed to answer these questions. Given that most previous studies on IRTree models did 
not conduct simulation studies to examine their statistical performance, it is extremely important to provide such evidence.

\section{Study 2. Simulation Study}

\section{Simulation Design}

We generated four types of four-point data sets that were either with or without the ERS and following either the unfolding or dominance response process. Specifically, the datasets without the ERS and following the dominance response process were generated from the SGRM with Equation 1 using R package mirt 1.33.2 (Chalmers, 2012). The datasets without the ERS and following the unfolding response process were generated from the GGUM as shown in Equation 3 using the R package GGUM 0.4.2 (Tendeiro \& Castro-Alvarez, 2019). The datasets with the ERS and following the dominance response process were generated from the DIRTree model as shown in Equations 4-10 using customized functions. The datasets with the ERS and following the unfolding response process were generated from the UIRTree model as shown in Equations 5-11 using customized functions.

The SGRM and the GGUM had the focal trait $\theta_{j}$ following a standard normal distribution $N(0,1)$ and discrimination parameters $\alpha_{i}$ following a uniform distribution unif $(0.5,2)$. In the SGRM, the distance between any consecutive location parameters $\delta_{i k}$ followed a uniform distribution unif $(0.3,1)$ and all location parameters were zero-centered. Location parameters $\delta_{i}$ in the GGUM followed a truncated standard normal distribution $N(0,1)$ with $-2<\delta_{i}<2$. As for threshold parameters in the GGUM, the R package GGUM 0.4.2 specified that $\tau_{i 0}=0$, and $\tau_{i 1}-\tau_{i 7}$ were constrained to symmetry around zero, that is, $\tau_{i 4}=0$ and $\tau_{i z}=-\tau_{i(8-z)}$ for $z \neq 0$. In the DIRTree model and the UIRTree model, the focal trait and the ERS followed a bivariate normal distribution, $\left(\theta_{j}, \zeta_{j}\right)^{\prime} \sim B V N(\boldsymbol{\mu}, \boldsymbol{\Sigma})$, where $\boldsymbol{\mu}=\mathbf{0}$, $\boldsymbol{\Sigma}=\left(\begin{array}{cc}\sigma_{\theta}{ }^{2} & \\ \sigma_{\theta} \sigma_{\zeta} \rho_{\theta \zeta} & \sigma_{\zeta}{ }^{2}\end{array}\right)$ with $\sigma_{\theta}^{2}=1$, and discrimination parameters $\alpha_{i}$ followed a uniform 
distribution unif $(0.5,2)$. Location parameters $\delta_{i}$ in the DIRTree model followed a uniform distribution unif $(-2,2)$, and location parameters $\delta_{i}$ in the UIRTree model had the same truncated standard normal distribution as that in the GGUM. Threshold parameters in the UIRTree model distributed as unif $(-2,-0.5)$. Lastly, in the DIRTree model and the UIRTree model, location parameters regarding the ERS, $\beta_{i 2}$ and $\beta_{i 3}$, were uniformly distributed as unif $(-2,2)$. Each generated dataset was then estimated with the SGRM, the GUMM, the DIRTree model, and the UIRTree model to check the model fit and parameter recovery. The number of items was fixed to 12 as it is a common length for personality measures (i.e., Brown \& Maydeu-Olivares, 2011; Cao et al., 2015; Soto \& John, 2017).

Three factors, sample size ( $N$; with three levels: $300,500,1,000)$, the variance of ERS $\left(\sigma_{\zeta}^{2}\right.$; with three levels: $\left.0.2,0.6,1.0\right)$, and the correlation between the ERS and the focal trait ( $\rho_{\theta \zeta}$; with three levels: $0,0.3,0.6$ ), were manipulated to check their influence on the performance of the estimation models. Since ERS was not involved in the datasets generated from the SGRM and the GGUM, we only examined the sample size for these two types of datasets. In total, there were three conditions of the datasets generated from the SGRM and the GGUM, and $3 \times 3 \times 3=27$ conditions of the datasets generated from the DIRTree model and the UIRTree model. The number of replications in each condition was 100. All simulated data sets were estimated by the R package mirt 1.33.2 (Chalmers, 2012) with the MMAP estimation method as in the empirical study.

It is expected that as $N$ increases, the correctly specified model (i.e., the model that is the same as the data generating model) and other models will have larger discrepancies regarding model fit and parameter recovery. Larger discrepancies between the models that account for ERS and the models that do not account for ERS are expected with the increase of $\sigma_{\zeta}^{2}$. As for $\rho_{\theta \zeta}$, Plieninger (2017) found that only when the correlation was non-zero could models ignoring the ERS lead to substantial bias in the scale reliability, but the effects of the 
correlation on model fit and parameter recovery were not studied. Accordingly, we looked into these aspects in this simulation study.

\section{Results}

Even though previous studies documented a substantial proportion of non-convergence when fitting the GGUM to data generated from the SGRM (e.g., Cao, Song, \& Tay, 2018), we note that all models, including those misspecified ones, converged properly in the present study. This is likely due to the fact that we incorporated priors for the estimation GGUM, which has been shown to be effective in reducing convergence issues and stabilizing estimates (de la Torre, Stark, \& Chernyshenko, 2006).

\section{Power of AIC and BIC for model selection}

The discrimination power (defined as the number of replications where the correctly specified model had the lowest AIC/BIC divided by the total number of replications in each condition) of AIC and BIC are shown in Table 4. AIC and BIC consistently had 100\% power to correctly distinguish the SGRM, the GGUM, and the DIRTree model across all conditions. When the data generating model is the UIRTree model, however, AIC and BIC no longer had perfect discrimination power as sometimes they wrongly preferred the DIRTree model.

As we can see, AIC consistently had very high discrimination power that was equal to or extremely close to 1 when the sample size $N$ was 500 or 1000 . When $N$ was as small as 300 , even though without perfect discrimination power, AIC still had the highest probability to correctly select the UIRTree model over other models. Furthermore, when the ERS variance $\sigma_{\zeta}^{2}$ was as sufficiently large as 1.0 (i.e., the same as the focal trait's variance), a higher $\rho_{\theta \zeta}$ would lead to better discrimination power of AIC for the UIRTree model.

The discrimination power of BIC for the UIRTree model was less satisfactory. In conditions with $N=300$, BIC almost always wrongly preferred the DIRTree model over the true UIRTree model. The poor discrimination power of BIC may result from the fact that more 
parameters are estimated in the UIRTree model compared to the DIRTree model, and the penalty of complexity was influential when the sample size was small. As $N$ increased, the discrimination power of BIC for the UIRTree model clearly improved. Besides, similar to AIC, BIC tended to have higher power as the latent correlation $\rho_{\theta \zeta}$ increased when $\sigma_{\zeta}^{2}=1$. When $N, \rho_{\theta \zeta}$, and ${\sigma_{\zeta}}^{2}$ were sufficiently large (in this simulation study, when $N=1000, \rho_{\theta \zeta}=0.6$, and $\sigma_{\zeta}^{2}=1$ ), the discrimination power of BIC approached as high as 0.93 .

\section{Table 4}

Discrimination power of AIC and BIC for the UIRTree model in the simulation study

\begin{tabular}{|c|c|c|c|c|c|c|}
\hline & \multicolumn{3}{|c|}{ Power of AIC } & \multicolumn{3}{|c|}{ Power of BIC } \\
\hline & $\mathrm{N}=300$ & $\mathrm{~N}=500$ & $\mathrm{~N}=1000$ & $\mathrm{~N}=300$ & $\mathrm{~N}=500$ & $\mathrm{~N}=1000$ \\
\hline & \multicolumn{6}{|c|}{$\sigma_{\zeta}^{2}=0.2$} \\
\hline$\rho_{\theta \zeta}=0.0$ & 0.78 & 0.97 & 1.00 & 0.02 & 0.23 & 0.69 \\
\hline$\rho_{\theta \zeta}=0.3$ & 0.78 & 0.94 & 1.00 & 0.03 & 0.24 & 0.74 \\
\hline \multirow[t]{2}{*}{$\rho_{\theta \zeta}=0.6$} & 0.84 & 0.97 & 1.00 & 0.05 & 0.25 & 0.79 \\
\hline & \multicolumn{6}{|c|}{$\sigma_{\zeta}^{2}=0.6$} \\
\hline$\rho_{\theta \zeta}=0.0$ & 0.83 & 0.98 & 0.99 & 0.04 & 0.2 & 0.73 \\
\hline$\rho_{\theta \zeta}=0.3$ & 0.82 & 0.93 & 1.00 & 0.02 & 0.28 & 0.71 \\
\hline \multirow[t]{2}{*}{$\rho_{\theta \zeta}=0.6$} & 0.93 & 0.99 & 1.00 & 0.07 & 0.48 & 0.89 \\
\hline & \multicolumn{6}{|c|}{$\sigma_{\zeta}^{2}=1.0$} \\
\hline$\rho_{\theta \zeta}=0.0$ & 0.72 & 0.95 & 1.00 & 0.02 & 0.15 & 0.68 \\
\hline$\rho_{\theta \zeta}=0.3$ & 0.81 & 0.95 & 1.00 & 0.02 & 0.25 & 0.67 \\
\hline$\rho_{\theta \zeta}=0.6$ & 0.92 & 0.97 & 1.00 & 0.07 & 0.37 & 0.93 \\
\hline
\end{tabular}

\section{Parameter recovery}

Parameter recovery was investigated based on mean bias (the mean of parameter estimates minus parameter true values), mean absolute bias (the mean of the absolute values of parameter estimates minus parameter true values), and root mean square error (RMSE; the square root of mean squared error of each parameter). The three indices were calculated for each parameter and then averaged across items. We focused on four aspects here. First, we examined whether model parameters could be accurately recovered when the correctly 
specified model was fitted to the data. Second, we examined parameter recovery of the SGRM and the GGUM when the data was generated from the DIRTree model and the UIRTree model, respectively. Third, we also examined item parameter recovery of the DIRTree model and the UIRTree model for datasets generated from the SGRM and the GGUM, respectively. Fourth, we looked into parameter recovery of the DIRTree model for datasets generated from the UIRTree model.

When the fitted model was correctly specified

The most important finding was that parameters for correctly specified UIRTree models could be estimated accurately across all conditions studied despite their complexity (see Table 5). Item parameters for correctly specified DIRTree models could also be estimated accurately across conditions. As expected, parameters for the SGRM and the GGUM were all accurately recovered as well even in conditions with only 300 respondents. 
Table 5

Parameter recovery for the four models as correctly specified models in the simulation study

\begin{tabular}{|c|c|c|c|c|c|c|c|c|c|}
\hline & \multicolumn{3}{|l|}{ Mean Bias } & \multicolumn{3}{|c|}{ Mean Absolute Bias } & \multicolumn{3}{|l|}{ RMSE } \\
\hline & Range & Mean & $\mathrm{SD}$ & Range & Mean & SD & Range & Mean & $\mathrm{SD}$ \\
\hline \multicolumn{10}{|c|}{ SGRM } \\
\hline$\alpha$ & {$[0.005,0.02]$} & 0.01 & 0.01 & {$[0.08,0.16]$} & 0.12 & 0.04 & {$[0.11,0.21]$} & 0.16 & 0.05 \\
\hline$\delta$ & {$[-0.01,0.01]$} & 0.002 & 0.01 & {$[0.07,0.15]$} & 0.11 & 0.03 & {$[0.09,0.19]$} & 0.13 & 0.04 \\
\hline \multicolumn{10}{|c|}{ GGUM } \\
\hline$\alpha$ & {$[-0.06,0.02]$} & -0.01 & 0.04 & {$[0.08,0.15]$} & 0.11 & 0.04 & {$[0.10,0.19]$} & 0.14 & 0.03 \\
\hline$\delta$ & {$[-0.05,0.07]$} & 0.01 & 0.06 & {$[0.04,0.09]$} & 0.07 & 0.03 & {$[0.06,0.12]$} & 0.1 & 0.03 \\
\hline$\tau$ & {$[-0.13,0.05]$} & -0.01 & 0.06 & {$[0.07,0.14]$} & 0.11 & 0.03 & {$[0.09,0.18]$} & 0.14 & 0.03 \\
\hline \multicolumn{10}{|c|}{ DIRTree model } \\
\hline$\alpha$ & {$[0.01,0.05]$} & 0.02 & 0.01 & {$[0.10,0.21]$} & 0.15 & 0.04 & {$[0.13,0.27]$} & 0.19 & 0.05 \\
\hline$\delta$ & {$[-0.01,0.01]$} & 0.001 & 0.004 & {$[0.07,0.14]$} & 0.11 & 0.03 & {$[0.09,0.18]$} & 0.13 & 0.03 \\
\hline$\sigma_{\zeta}$ & {$[-0.01,0.02]$} & 0.003 & 0.01 & {$[0.02,0.06]$} & 0.04 & 0.01 & {$[0.02,0.06]$} & 0.04 & 0.01 \\
\hline$\beta_{2}$ & {$[-0.01,0.02]$} & -0.001 & 0.01 & {$[0.09,0.18]$} & 0.14 & 0.03 & {$[0.12,0.23]$} & 0.17 & 0.04 \\
\hline$\beta_{3}$ & {$[-0.01,0.01]$} & 0.0003 & 0.01 & {$[0.09,0.18]$} & 0.13 & 0.03 & {$[0.11,0.23]$} & 0.17 & 0.04 \\
\hline$\rho_{\theta \zeta}$ & {$[-0.01,0.03]$} & 0.001 & 0.01 & {$[0.02,0.10]$} & 0.05 & 0.02 & {$[0.02,0.10]$} & 0.05 & 0.02 \\
\hline \multicolumn{10}{|c|}{ UIRTree model } \\
\hline$\alpha$ & {$[-0.01,0.01]$} & -0.005 & 0.01 & {$[0.12,0.22]$} & 0.18 & 0.04 & {$[0.16,0.28]$} & 0.22 & 0.05 \\
\hline$\delta$ & {$[-0.03,0.03]$} & -0.001 & 0.01 & {$[0.12,0.26]$} & 0.19 & 0.05 & {$[0.17,0.35]$} & 0.26 & 0.06 \\
\hline$\tau$ & {$[0.02,0.09]$} & 0.05 & 0.02 & {$[0.11,0.23]$} & 0.17 & 0.04 & {$[0.16,0.33]$} & 0.24 & 0.06 \\
\hline$\sigma_{\zeta}$ & {$[-0.01,0.01]$} & 0.002 & 0.01 & {$[0.02,0.05]$} & 0.04 & 0.01 & {$[0.02,0.05]$} & 0.04 & 0.01 \\
\hline$\beta_{2}$ & {$[-0.01,0.01]$} & -0.001 & 0.01 & {$[0.10,0.20]$} & 0.14 & 0.04 & {$[0.12,0.25]$} & 0.18 & 0.04 \\
\hline$\beta_{3}$ & {$[-0.01,0.01]$} & -0.001 & 0.01 & {$[0.08,0.16]$} & 0.12 & 0.03 & {$[0.10,0.20]$} & 0.14 & 0.04 \\
\hline$\rho_{\theta \zeta}$ & {$[-0.02,0.12]$} & 0.02 & 0.03 & {$[0.03,0.12]$} & 0.06 & 0.02 & {$[0.03,0.12]$} & 0.06 & 0.02 \\
\hline
\end{tabular}

Note. The means and SDs of parameter recovery indices were computed across all conditions.

\section{When the ERS was ignored}

It was found in the empirical study that discrimination parameters on the focal trait, $\alpha$, had smaller values in the GGUM compared to the UIRTree model and in the SGRM compared to the DIRTree model. This pattern was also observed in this simulation study. For datasets generated from the UIRTree model (the DIRTree model), estimates of $\alpha$ from the GGUM (the SGRM) had mean bias ranging from -0.70 to -0.61 (from -0.45 to -0.39 ), mean absolute bias 
ranging from 0.61 to 0.70 (from 0.41 to 0.45 ), and RMSE ranging from 0.66 to 0.76 (from 0.46 to 0.52 ). The negative mean bias suggested that ignoring response ERS led to underestimated the discrimination ability of items. Furthermore, the finding that the sizes of mean bias and mean absolute bias were similar suggested that the underestimation occurred in almost all replications. No clear effects of $N, \sigma_{\zeta}^{2}$, and $\rho_{\theta \zeta}$ on the bias of $\alpha$ were found.

In line with the empirical findings, estimates of location parameters regarding the focal trait, $\delta$, had more extreme values in the GGUM compared to the UIRTree model for intermediate items. As we can see in Figure 4, there seemed to be an interaction between $\sigma_{\zeta}^{2}$ and $\rho_{\theta \zeta}$ on the three parameter recovery indices of $\delta$ in the GGUM when estimating datasets generated from the UIRTree model. When the ERS and the focal trait were uncorrelated (i.e., $\rho_{\theta \zeta}=0$ ), the parameter recovery of $\delta$ in the GGUM was relatively small regardless of the value of $\sigma_{\zeta}^{2}$; when the ERS and the focal trait were correlated (i.e., $\rho_{\theta \zeta} \neq 0$ ), the parameter recovery of $\delta$ in the GGUM became clearly worse and increased with larger $\sigma_{\zeta}{ }^{2}$. The increase of $N$ seemed to have no influence on the mean bias of $\delta$ while it did decrease its mean absolute bias and the RMSE. Results from linear regression analysis also revealed a significant interaction effect between $\sigma_{\zeta}^{2}$ and $\rho_{\theta \zeta}$ on all of the three parameter recovery indices of $\delta$ and a significantly negative main effect of $N$ on mean absolute bias and RMSE of $\delta$. 

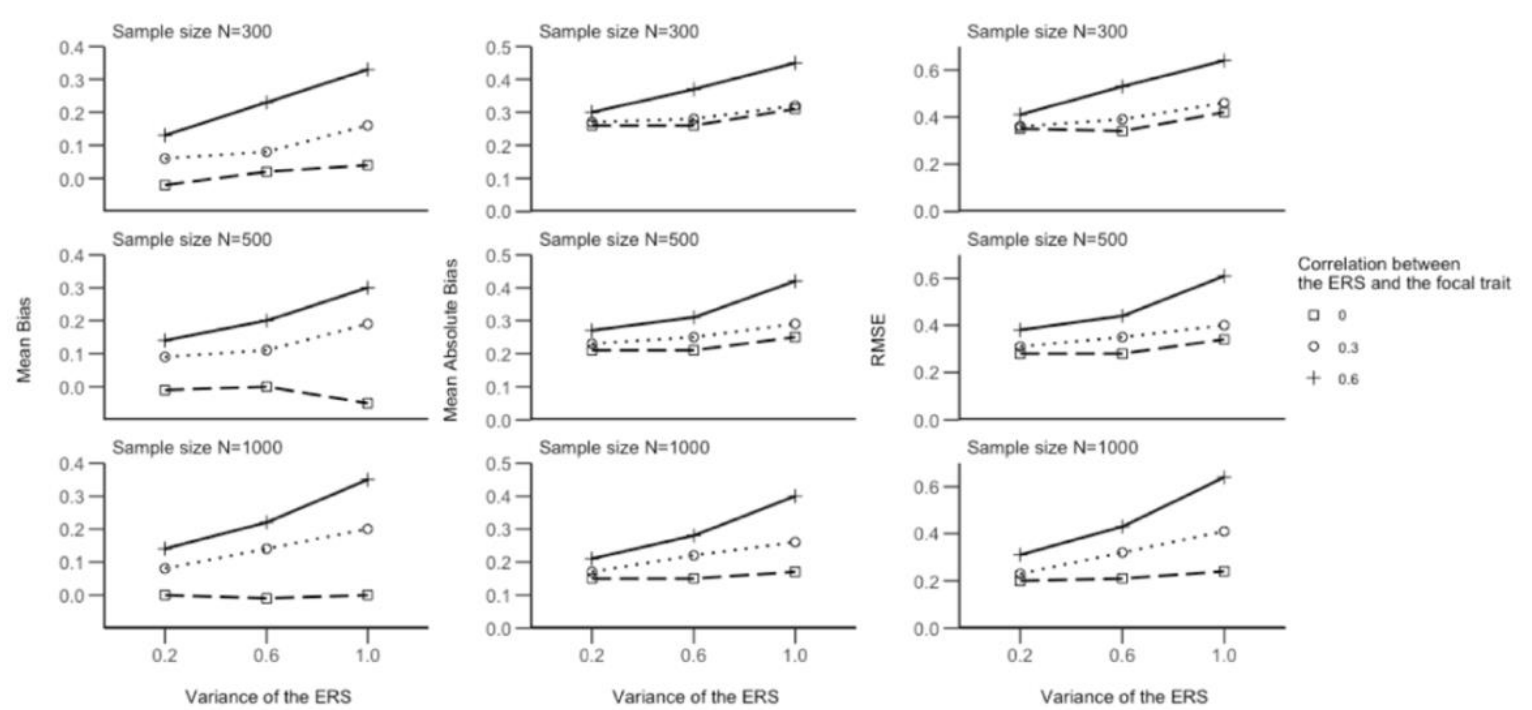

Figure 4. Parameter recovery of $\boldsymbol{\delta}$ in the GGUM for datasets generated from the UIRTree model

When the ERS was falsely assumed

When the dataset was generated from the GGUM, the discrimination parameters of the UIRTree model had mean bias of $0.62,0.82$, and 0.92 , mean absolute bias of $0.62,0.82$, and 0.92 , and RMSE of $0.65,0.87$, and 0.96 in conditions with sample size $N$ equal to 300,500 , and 1000, respectively, indicating that the UIRTree model consistently overestimated $\alpha$. Moreover, the absence of the ERS did not distort the estimation of $\delta$ in the UIRTree model substantially, with mean bias of $0.07,-0.05$, and 0.01 , mean absolute bias of $0.14,0.09$, and 0.05 , and RMSE of $0.18,0.10$, and 0.07 . For datasets generated from the SGRM, the DIRTree model brought about mean bias of $0.03,0.02$, and 0.004 , mean absolute bias of $0.20,0.15$, and 0.10 , and RMSE of $0.26,0.19$, and 0.13 for $\alpha$, suggesting that falsely assuming the ERS would not substantially impact the estimation of discrimination parameters on the focal trait.

When the dominance response process was falsely assumed

Lastly, we investigated parameter recovery of the DIRTree model for datasets generated from the UIRTree model. The common parameters of the two models were the variance of the ERS $\sigma_{\zeta}^{2}$, location parameters regarding the ERS $\beta_{2}$ and $\beta_{3}$, and the latent correlation between 
the ERS and the focal trait $\rho_{\theta \zeta}$. Since the nodes for the ERS were coded and modeled the same for the two IRTree models, the parameter recovery of $\sigma_{\zeta}^{2}, \beta_{2}$, and $\beta_{3}$ of the DIRTree model, as expected, were satisfactory with mean bias ranging from -0.01 to 0.01 , mean absolute bias ranging from 0.02 to 0.20 , and RMSE ranging from 0.02 to 0.24 . These parameter recovery indices were close to those of the UIRTree model. Surprisingly. $\rho_{\theta \zeta}$ was sufficiently accurately estimated such that the mean bias ranged from -0.11 to 0.11 , the mean absolute bias ranged from 0.04 to 0.17 , and the RMSE ranged from 0.04 to 0.17 in the DIRTree model.

\section{General Discussion}

Correctly accounting for item response process and RSs are of fundamental importance in psychological measurement. However, previous research on item response process and RSs has been growing largely in parallel. The present study aimed to integrate the unfolding response process and RSs in a single modeling framework by proposing the UIRTree model. The empirical illustration showed that respondents were likely to have followed an unfolding response process, and the ERS had a strong presence. Monte Carlo simulations further showed that (1) model parameters in the UIRTree model could be accurately recovered under realistic conditions, (2) AIC was very powerful in selecting among competing models, (3) BIC was biased towards the DIRTree model (i.e., the simpler model) versus the UIRTree model in conditions with relatively small sample size, small ERS magnitude, and/or low correlation between the focal trait and the ERS, and (4) wrongly assuming the item response process or ignoring RSs was detrimental to the estimation of key parameters. Overall, the current study presented a flexible framework that is expected to help researchers better reveal how respondents respond to non-cognitive items.

\section{Model Selection}

We investigated the power of AIC and BIC to select among competing models and found that AIC was very effective in the absolute sense while BIC was biased towards the 
DIRTree model when the correctly specified model was the UIRTree model. This finding is very important in that it tells us to have more confidence in AIC instead of BIC when choosing between the DIRTree and UIRTree models. We suspect that BIC's heavy penality for model complexity is the reason for the weaker power of BIC compared to AIC. Compared with the DIRTree model, the UIRTree model has two more parameters per item. In the current simulation, the fitted UIRTree model had 24 more parameters than the DIRTree model. However, the item response curve of a dominance model can overlap with a substantial proportion of that of an unfolding model depending on the location of the item (see Figure 1 in Tay et al., 2011). The further away an item is from zero, the more overlap. Therefore, the degree of model-data misfit might not be as dramatic as we would expect because item locations spanned from -2 to 2 . The moderate degree of misfit might be countered by BIC's penalty for the 24 additional parameters, thus leading to the low power of BIC.

\section{Parameter recovery for the UIRTree model}

It is reassuring to see that key parameters in the UIRTree model can be well recovered even in conditions with only 300 respondents. Earlier studies on the estimation of the GGUM showed that a sample size of 750 would be a requirement for reasonably accurate estimates when a marginal maximum likelihood estimator was used (Roberts et al., 2000). Otherwise, researchers are likely to obtain some wild estimates (de la Torre et al., 2006). In the current study, moderately informative priors were used for discrimination parameters and location parameters to softly constrain them parameters within a reasonable range. Our simulation showed that the incorporation of priors into the estimation process greatly reduced sample size requirement. Future users of the UIRTree model (or GGUM) are strongly encouraged to use such priors for more stable and accurate estimates.

\section{Implications}


This study has both theoretical and practical implications. Theoretically, we proposed an integrative modeling framework that can simultaneously account for the unfolding response process and RSs. This modeling framework has the potential to help researchers better understand the cognitive processes underlying item responding, which is critical for measurement validity (Borsboom et al., 2004). Practically, the UIRTree model may be helpful for scale development. It is known that writing intermediate items is difficult because a seemingly good item often turns out to have lower discrimination parameters or more extreme location parameters than expected when analyzed with the GGUM. Our simulation results showed that low discrimination parameters or more extreme location parameters might result from unmodeled ERS. Of course, it should be noted that falsely assuming ERS will lead to overestimated discrimination parameters (but location parameters were not biased). Therefore, for scales that include intermediate items or where intermediate are suspected to exist, researchers should first fit the GGUM and the UIRTree model, use AIC to compare model fit, and then check the magnitude of the ERS. If AIC selects the GGUM and the magnitude of the ERS is small, then results from the GGUM are more trustworthy. If AIC prefers the UIRTree and the magnitude of the ERS is substantial, researchers should trust results from the UIRTree model more. Moreover, this study also provides tutorials for estimating the UIRTree model, which allows the model to be more accessible for practitioners.

\section{Limitation and future directions}

Despite the merits discussed above, the current study is still limited in the following aspects. First, the UIRTree model proposed here only accounted for a single focal trait. Future researchers are encouraged to extend the current one to a multi-trait UIRTree model. One additional advantage of that multi-trait UIRTree model is that we could formally test whether a certain type of RS is generalizable across constructs or not (Zhang, Luo et al., 2021). However, caution should be exercised in model estimation since traditional estimation methods for item 
response models usually have trouble handling high-dimensional structures (Wirth \& Edwards, 2007). Second, the current model implicitly assumes population heterogeneity such that the same model applies to everyone. However, studies suggested that different people may follow different item response processes, and some people may not engage in extreme responding while others do (Tijmstra, Bolsinova, \& Jeon, 2018). Therefore, future researchers could develop some mixture models to account for such population heterogeneity. Third, other types of RSs apart from the ERS were not examined. Given the flexibility of the UIRTree model, future researchers are encouraged to extend the current version to include more than one type of RSs.

\section{Conclusion}

With evidence from both the empirical illustration and the simulation study, the proposed UIRTree model shows potential in estimating responses with unfolding response processes and influenced by RSs. Incorrectly specifying response process or ignoring RSs is likely to cause estimation bias and thus distort measurement and item selection procedures. Researchers are encouraged to consider the UIRTree model when modeling Likert-scale responses. 


\section{References}

Ames, A. J., \& Myers, A. J. (2020). Explaining variability in response style traits: A covariate-adjusted IRTree. Educational and Psychological Measurement. Advance online publication. https://doi.org/10.1177/0013164420969780

Anglim, J., Horwood, S., Smillie, L. D., Marrero, R. J., \& Wood, J. K. (2020). Predicting psychological and subjective well-being from personality: A metaanalysis. Psychological Bulletin, 146(4), 279323. https://doi.org/10.1037/bu10000226

Batchelor, J. H., \& Miao, C. (2016). Extreme response style: A meta-analysis. Journal of Organizational Psychology, 16(2), 51-62. Retrieved from https://articlegateway.com/index.php/JOP/article/view/1790

Baumgartner, H., \& Steenkamp, J. B. E. (2001). Response styles in marketing research: A cross-national investigation. Journal of marketing research, 38(2), 143-156. https://doi.org/10.1509/jmkr.38.2.143.18840

Böckenholt, U. (2012). Modeling multiple response processes in judgment and choice. Psychological Methods, 17, 665-678. https://doi.org/10.1037/2325-9965.1.S.83

Böckenholt, U. (2017). Measuring response styles in Likert items. Psychological Methods, 22(1), 69-83. https://doi.org/10.1037/met0000106

Böckenholt, U. (2019). Assessing item-feature effects with item response tree models. British Journal of Mathematical and Statistical Psychology, 72(3), 486500. https://doi.org/10.1111/bmsp.12163

Borsboom, D., Mellenbergh, G. J., \& van Heerden, J. (2004). The Concept of Validity. Psychological Review, 111(4), 1061-1071. https://doi.org/10.1037/0033295X.111.4.1061 
Brown, A., \& Maydeu-Olivares, A. (2011). Item response modeling of forced-choice questionnaires. Educational and Psychological Measurement, 71(3), 460-502. https://doi.org/10.1177/0013164410375112

Cao, M., Drasgow, F., \& Cho, S. (2015). Developing ideal intermediate personality items for the ideal point model. Organizational Research Methods, 18(2), 252-275. https://doi.org/10.1177/1094428114555993

Cao, M., Song, Q. C., \& Tay, L. (2018). Detecting curvilinear relationships: a comparison of scoring approaches based on different item response models. International Journal of Testing, 18(2), 178-205. https://doi.org/10.1080/15305058.2017.1345913

Carter, N. T., \& Dalal, D. K. (2010). An ideal point account of the JDI Work satisfaction scale. Personality and Individual Differences, 49(7), 743-748. https://doi.org/10.1016/j.paid.2010.06.019

Carter, N. T., Dalal, D. K., Boyce, A. S., O'Connell, M. S., Kung, M.-C., \& Delgado, K. M. (2014). Uncovering curvilinear relationships between conscientiousness and job performance: How theoretically appropriate measurement makes an empirical difference. Journal of Applied Psychology, 99(4), 564-586. http://dx.doi.org/10.1037/a0034688

Carter, N. T., Dalal, D. K., Guan, L., LoPilato, A. C., \& Withrow, S. A. (2017). Item response theory scoring and the detection of curvilinear relationships. Psychological Methods, 22(1), 191-203. https://doi.org/10.1037/met0000101

Chalmers, R. P. (2012). mirt: A multidimensional item response theory package for the R environment. Journal of statistical Software, 48(1), 1-29. https://doi.org/10.18637/jss.v048.i06

Chernyshenko, O. S., Stark, S., Drasgow, F., \& Roberts, B. W. (2007). Constructing personality scales under the assumptions of an ideal point response process: Toward 
increasing the flexibility of personality measures. Psychological Assessment, 19(1), 88-106. https://doi.org/10.1037/1040-3590.19.1.88

Cho, S., Drasgow, F., \& Cao, M. (2015). An investigation of emotional intelligence measures using item response theory. Psychological Assessment, 27(4), 12411252. https://doi.org/10.1037/pas0000132

De Boeck, P., \& Partchev, I. (2012). IRTrees: Tree-based item response models of the GLMM family. Journal of Statistical Software, 48(1), 1-28. https://doi.org/10.18637/jss.v048.c01

De la Torre, J., Stark, S., \& Chernyshenko, O. S. (2006). Markov chain Monte Carlo estimation of item parameters for the generalized graded unfolding model. Applied Psychological Measurement, 30(3), 216-232. https://doi.org/10.1177/0146621605282772

Drasgow, F., Chernyshenko, O., \& Stark, S. (2010). 75 Years After Likert: Thurstone Was Right! Industrial and Organizational Psychology, 3(4), 465-476. https://doi.org/10.1111/j.1754-9434.2010.01273.x

Drasgow, F., Stark, S., Chernyshenko, O. S., Nye, C. D., Hulin, C. L., \& White, L. A. (2012). Development of the tailored adaptive personality assessment system (TAPAS) to support army personnel selection and classification decisions. Drasgow Consulting Group Urbana IL.

Eid, M., \& Rauber, M. (2000). Detecting measurement invariance in organizational surveys. European Journal of Psychological Assessment, 16(1), 2030. https://doi.org/10.1027/1015-5759.16.1.20

Feintzeig, R. (2015), “I don’t have a job, I have a higher calling”, The Wall Street Journal, 24 February. 
Henninger, M., \& Meiser, T. (2020). Different approaches to modeling response styles in divide-by-total item response theory models (part 1): A model integration. Psychological Methods, 25(5), 560576. https://doi.org/10.1037/met0000249

Javaras, K. N., \& Ripley, B. D. (2007). An “unfolding” latent variable model for Likert attitude data: Drawing inferences adjusted for response style. Journal of the American Statistical Association, 102(478), 454-463.

https://doi.org/10.1198/016214506000000960

Jeon, M., \& De Boeck, P. (2016). A generalized item response tree model for psychological assessments. Behavior research methods, 48(3), 1070-1085. https://doi.org/10.3758/s13428-015-0631-y

Johnson, T. R., \& Bolt, D. M. (2010). On the use of factor-analytic multinomial logit item response models to account for individual differences in response style. Journal of Educational and Behavioral Statistics, 35(1), 92-114.

https://doi.org/10.3102/1076998609340529

Judge, T. A., Rodell, J. B., Klinger, R. L., Simon, L. S., \& Crawford, E. R. (2013). Hierarchical representations of the five-factor model of personality in predicting job performance: Integrating three organizing frameworks with two theoretical perspectives. Journal of Applied Psychology, 98(6), 875925. https://doi.org/10.1037/a0033901

Lang, J. W. B., Lievens, F., De Fruyt, F., Zettler, I., \& Tackett, J. L. (2019). Assessing meaningful within-person variability in Likert-scale rated personality descriptions: An IRT tree approach. Psychological Assessment, 31(4), 474487. https://doi.org/10.1037/pas0000600 
Lang, J. W., \& Tay, L. (2021). The science and practice of item response theory in organizations. Annual Review of Organizational Psychology and Organizational Behavior, 8, 311-338. https://doi.org/10.1146/annurev-orgpsych-012420-061705

Lievens, F., Lang, J. W. B., De Fruyt, F., Corstjens, J., Van de Vijver, M., \& Bledow, R. (2018). The predictive power of people's intraindividual variability across situations: Implementing whole trait theory in assessment. Journal of Applied Psychology, 103(7), 753-771. https://doi.org/10.1037/ap10000280

Ling, Y., Zhang, M., Locke, K. D., Li, G., \& Li, Z. (2016). Examining the process of responding to Circumplex Scales of Interpersonal Values items: should ideal point scoring methods be considered?. Journal of personality assessment, 98(3), 310-318. https://doi.org/10.1080/00223891.2015.1077852

Liu, C. W., \& Wang, W. C. (2018). A general unfolding IRT model for multiple response styles. Applied psychological measurement, 43(3), 195-210. https://doi.org/10.1177/0146621618762743

McDaniel, M. A., Psotka, J., Legree, P. J., Yost, A. P., \& Weekley, J. A. (2011). Toward an understanding of situational judgment item validity and group differences. Journal of Applied Psychology, 96(2), 327-336. https://doi.org/10.1037/a0021983

Moors, G. (2004). Facts and artefacts in the comparison of attitudes among ethnic minorities. A multigroup latent class structure model with adjustment for response style behavior. European Sociological Review, 20(4), 303-320. https://doi.org/10.1093/esr/jch026

Muraki, E. (1992). A generalized partial credit model: Application of an EM algorithm. Applied Psychological Measurement, 16, 159-176. https://doi.org/10.1002/j.23338504.1992.tb01436.x 
Nye, C. D., Prasad, J., \& Rounds, J. (2021). The effects of vocational interests on motivation, satisfaction, and academic performance: Test of a mediated model. Journal of Vocational Behavior, 127, 103583. https://doi.org/10.1016/j.jvb.2021.103583

Nye, C. D., Su, R., Rounds, J., \& Drasgow, F. (2012). Vocational interests and performance: A quantitative summary of over 60 years of research. Perspectives on Psychological Science, 7(4), 384-403. https://doi.org/10.1177/1745691612449021

Organisation for Economic Co-operation and Development (2018): Programme for the International Assessment of Adult Competencies (PIAAC), International Pilot Study on Non-Cognitive Skills. GESIS Data Archive, Cologne. ZA6941 Data file Version 1.0.0, https://doi.org/10.4232/1.13063

Plieninger, H. (2017). Mountain or molehill? A simulation study on the impact of response styles. Educational and Psychological Measurement, 77(1), 32-53. https://doi.org/10.1177/0013164416636655

Poropat, A. E. (2009). A meta-analysis of the five-factor model of personality and academic performance. Psychological Bulletin, 135(2), 322338. https://doi.org/10.1037/a0014996

Rasch, G. (1960). Probabilistic models for some intelligence and attainment tests. Copenhagen: Danish Institute for Educational Research.

Roberts, J. S., \& Thompson, V. M. (2011). Marginal maximum a posteriori item parameter estimation for the generalized graded unfolding model. Applied Psychological Measurement, 35(4), 259-279. https://doi.org/10.1177/0146621610392565

Roberts, J. S., Donoghue, J. R., \& Laughlin, J. E. (2000). A general item response theory model for unfolding unidimensional polytomous responses. Applied Psychological Measurement, 24(1), 3-32. https://doi.org/10.1177/01466216000241001 
Roberts, J. S., Fang, H.-r., Cui, W., \& Wang, Y. (2006). GGUM2004: A Windows-Based Program to Estimate Parameters in the Generalized Graded Unfolding Model. Applied Psychological Measurement, 30(1), 64-

65. https://doi.org/10.1177/0146621605280141

Roberts, J. S., Laughlin, J. E., \& Wedell, D. H. (1999). Validity issues in the Likert and Thurstone approaches to attitude measurement. Educational and psychological measurement, 59(2), 211-233. https://doi.org/10.1177/00131649921969811

Rorer, L. G. (1965). The great response-style myth. Psychological Bulletin, 63(3), 129156. https://doi.org/10.1037/h0021888

Samejima, F. (1969). Estimation of latent ability using a response pattern of graded scores. Psychometrika Monograph Supplement, 34(4, Pt. 2), 100. https://doi.org/10.1002/j.2333-8504.1968.tb00153.x

Soto, C. J., \& John, O. P. (2017). The next Big Five Inventory (BFI-2): Developing and assessing a hierarchical model with 15 facets to enhance bandwidth, fidelity, and predictive power. Journal of Personality and Social Psychology, 113(1), 117143. https://doi.org/10.1037/pspp0000096

Stark, S., Chernyshenko, O. S., Drasgow, F., \& Williams, B. A. (2006). Examining assumptions about item responding in personality assessment: Should ideal point methods be considered for scale development and scoring? Journal of Applied Psychology, 91(1), 25-39. https://doi.org/10.1037/0021-9010.91.1.25

Sun, T., Zhang, B., Cao, M., \& Drasgow, F. (2021). Faking detection improved: Adopting a Likert item response process tree model. Organizational Research Methods. https://doi.org/10.1177/10944281211002904

Tay, L., \& Drasgow, F. (2012). Theoretical, statistical, and substantive issues in the assessment of construct dimensionality: Accounting for the item response 
process. Organizational Research Methods, 15(3), 363-384.

https://doi.org/10.1177/1094428112439709

Tay, L., \& Ng, V. (2018). Ideal point modeling of non-cognitive constructs: Review and recommendations for research. Frontiers in psychology, 9, 2423. https://doi.org/10.3389/fpsyg.2018.02423

Tay, L., Ali, U. S., Drasgow, F., \& Williams, B. (2011). Fitting IRT models to dichotomous and polytomous data: Assessing the relative model-data fit of ideal point and dominance models. Applied Psychological Measurement, 35(4), 280-295. https://doi.org/10.1177/0146621610390674

Tay, L., Drasgow, F., Rounds, J., \& Williams, B. A. (2009). Fitting measurement models to vocational interest data: Are dominance models ideal? Journal of Applied Psychology, 94(5), 1287-1304. https://doi.org/10.1037/a0015899

Tendeiro, J. N., \& Castro-Alvarez, S. (2019). GGUM: An R package for fitting the generalized graded unfolding model. Applied psychological measurement, 43(2), 172 173. https://doi.org/10.1177/0146621618772290

Tijmstra, J., Bolsinova, M., \& Jeon, M. (2018). General mixture item response models with different item response structures: Exposition with an application to Likert scales. Behavior research methods, 50(6), 2325-2344. https://doi.org/10.3758/s13428017-0997-0

Tu, N., Zhang, B., Angrave, L. C., \& Sun, T. (2021). bmggum: An R package for Bayesian estimation of Multidimensional Generalized Graded Unfolding Model with covariates. Applied Psychological Measurement. Advanced online publication.

Wetzel, E., Lüdtke, O., Zettler, I., \& Böhnke, J. R. (2016). The stability of extreme response style and acquiescence over 8 years. Assessment, 23(3), 279-291. https://doi.org/10.1177/1073191115583714 
Wirth, R. J., \& Edwards, M. C. (2007). Item factor analysis: Current approaches and future directions. Psychological Methods, 12(1), 58-79. https://doi.org/10.1037/1082989X.12.1.58

Zettler, I., Lang, J. W., Hülsheger, U. R., \& Hilbig, B. E. (2016). Dissociating indifferent, directional, and extreme responding in personality data: Applying the three-process model to self-and observer reports. Journal of Personality, 84(4), 461-472. https://doi.org/10.1111/jopy.12172

Zhang, B., Cao, M., Tay, L., Luo, J., \& Drasgow, F. (2020). Examining the item response process to personality measures in high-stakes situations: Issues of measurement validity and predictive validity. Personnel Psychology, 73(2), 305-332. https://doi.org/10.1111/peps.12353

Zhang, B., Luo, J., Sun, T., Cao, M., \& Drasgow, F. (2021). Small but Nontrivial: A Comparison of Six Strategies to Handle Cross-Loadings in Bifactor Predictive Models. Multivariate Behavioral Research, 1-18.

https://doi.org/10.1080/00273171.2021.1957664 